\title{
Glucose Transporter 4 and Peroxisome Proliferator- Activated Receptor-Alpha Overexpression Association With Cardioprotective Effects of Myoinositol and Metformin Combination in Type 2 Diabetic Rat Model
}

\author{
Dalia Abdel-Wahab Mohamed ${ }^{\mathrm{a}, \mathrm{g}}$, Wael M. Elayat ${ }^{\mathrm{a}}$, Asmaa A. Abozeid ${ }^{\mathrm{b}, \mathrm{c}}$, \\ Mohamed F. Abdel-Salam ${ }^{\text {, Nahla Mohamed Teama }}{ }^{\mathrm{e}}$, \\ Dina Sayed Abdelrahim ${ }^{\mathrm{f}}$, Yomna M. Tamim ${ }^{\mathrm{f}}$
}

\begin{abstract}
Background: Type 2 diabetes mellitus (DM) has many complications associated with increased morbidity and mortality. Cardiovascular complications are one of these serious complications. Therefore, there is a need for early and effective management to hamper them. This study aimed to evaluate the cardioprotective efficacy of metformin and myoinositol combination through exploring the associated underlying mechanisms in type $2 \mathrm{DM}$ rat model.

Methods: Type 2 DM model was induced in rats by streptozotocin and high-fat diet. Rats were treated with metformin $(150 \mathrm{mg} / \mathrm{kg})$ and $/$ or myoinositol $(50 \mathrm{mg} / \mathrm{kg})$ by oral gavage once daily for 4 weeks. Immunohistochemistry was used to assess the drugs' cardioprotective efficacy by estimating troponin $\mathrm{T}$, nuclear factor kappa B (NF-кB) and tumor necrosis factor alpha $(\mathrm{TNF}-\alpha)$. Quantitative real-time polymerase chain reaction and enzyme-linked immunosorbent assay were utilized to investigate peroxisome proliferator-activated receptor-alpha (PPAR $\alpha$ ) and glucose transporter 4 (GLUT-4) expression levels in the skeletal muscles, respectively.
\end{abstract}

Results: This study found that metformin and myoinositol combi-

Manuscript submitted July 31, 2021, accepted October 4, 2021

Published online October 31, 2021

aDepartment of Medical Biochemistry and Molecular Biology, Faculty of Medicine, Ain Shams University, PO Box 11381, Cairo, Egypt

bepartment of Histology, Faculty of Medicine, Ain Shams University, PO Box 11381, Cairo, Egypt

${ }^{\mathrm{c}}$ Armed Forces College of Medicine (AFCM), Cairo, Egypt

dDepartment of Physiology, Faculty of Medicine, Ain Shams University, PO Box 11381, Cairo, Egypt

eDepartment of Internal Medicine and Nephrology, Faculty of Medicine, Ain Shams University, PO Box 11381, Cairo, Egypt

fDepartment of Pharmacology, Faculty of Medicine, Ain Shams University, PO Box 11381, Cairo, Egypt

'Corresponding Author: Dalia Abdel-Wahab Mohamed, Department of Medical Biochemistry, Faculty of Medicine, Ain-Shams University, Cairo, Egypt. Email: dalia_wahab@med.asu.edu.eg

doi: https://doi.org/10.14740/jem765 nation was associated with GLUT-4 and PPAR $\alpha$ overexpression, together with better lipid profile than metformin alone in diabetic rats. Additionally, the combination of both drugs improved troponin $\mathrm{C}$ and decreased creatine kinase MB isoenzyme, NF- $\mathrm{kB}$ and TNF- $\alpha$ cardiac levels.

Conclusion: The current study indicated that myoinositol in combination with metformin had better cardioprotective effect than metformin alone in type $2 \mathrm{DM}$. This favorable effect was exhibited through upregulation of GLUT-4 and PPAR $\alpha$ receptors expression in skeletal muscles, thus increasing insulin sensitivity and improving lipid profile.

Keywords: Type 2 diabetes mellitus; Metformin; Myoinositol; PPAR $\alpha$; GLUT-4; NF-кB; TNF- $\alpha$

\section{Introduction}

Diabetes mellitus (DM) is a group of metabolic diseases characterized by hyperglycemia resulting from defects in insulin secretion, insulin action, or both. It is associated with microvascular and macrovascular complications, including retinopathy, nephropathy, and neuropathy, as well as cerebrovascular disease, ischemic heart disease, and peripheral artery disease [1]. Type $2 \mathrm{DM}$ is the most prevalent type, representing 90$95 \%$ of DM and occurs due to a progressive loss of $\beta$-cell insulin secretion frequently on the background of insulin resistance [2].

DM is related with multiple cardiovascular complications including atherosclerosis, stroke, ischemic heart disease and peripheral vascular disease. It is also associated with cardiomyopathy which is one of the most frequent and common chronic complication that happens in type 2 DM [3]. It occurs in $12 \%$ of the diabetic patients leading to heart failure and death. Nearly one half of all deaths in diabetic patients are due to cardio-vascular diseases [4]. Therefore, one of the targets in diabetes treatment is controlling blood glucose level, thus preventing various cardiovascular insults. The management goals 
for type 2 DM are to prevent or delay complications and maintain quality of life. This requires early diagnosis and treatment of hyperglycemia in addition to management of cardiovascular risk factors [5]. Although the main pathogenesis of type 2 DM is insulin resistance, there are only two of all antidiabetic drugs (thiazolidinedione and metformin) that act by increasing insulin sensitivity [2].

Glucose transporter 4 (GLUT-4) is a major mediator of glucose removal from the circulation and a key regulator of whole-body glucose homeostasis. It is highly expressed in adipose tissue and skeletal muscle. Decrease in GLUT-4 expression and/or decrease in its translocation in the skeletal muscle and adipose tissue lead to insulin resistance and propensity toward DM [6]. This peripheral insulin resistance causes pancreatic $\beta$ cells to secrete more insulin, in a process known as compensatory hyperinsulinemia. However, together with insulin resistance, often there is $\beta$-cell depletion, which results in sustained hyperglycemia and type 2 DM [7].

Peroxisome proliferator-activated receptors (PPARs) are ligand activated transcription factors regulating important genes in cell differentiation and various metabolic processes, particularly lipid and glucose homeostasis [8]. The PPARs family consists of three isoforms: $\operatorname{PPAR} \alpha, \operatorname{PPAR} \beta / \delta$ and $\operatorname{PPAR} \gamma$ that differ from each other in tissue distributions, ligand specificities and physiological roles [9].

PPAR $\alpha$ is highly expressed in metabolically active tissues, such as liver, heart, skeletal muscle, intestinal mucosa, and brown adipose tissue. This receptor is involved in fatty acid metabolism and its activation lowers lipid levels [10]. It also has a role in glucose homeostasis and insulin resistance development [11]. In the liver, PPAR $\alpha$ stimulates gluconeogenesis and ketone body synthesis and is involved in lipoprotein assembly. In the heart, it participates in the regulation of the metabolic switch between glucose and lipid oxidation [12]. This led to the development of many synthetic PPARs agonists for the treatment of different clinical outcomes over the past several decades [13]. For example, PPAR $\alpha$ activators such as fibrates (fenofibrate and clofibrate) are useful drugs for the treatment of dyslipidemia through increasing high-density lipoprotein (HDL) and decreasing triglycerides (TGs) with no effects on low-density lipoprotein (LDL). PPAR $\gamma$ is a target of synthetic insulin sensitizers thiazolidinediones such as pioglitazone and rosiglitazone, which are used in the treatment of type 2 DM. Dual agonists of PPAR $\alpha / \gamma$, such as glitazar, have been developed and have recently become available for the combined treatment of type 2 DM and dyslipidemia [14].

The underlying pathophysiological features and subcellular mechanisms responsible for the development of diabetic cardiomyopathy are not completely understood. Metabolic abnormalities and overproduction of reactive oxygen and nitrogen species may trigger various intracellular pathways and alter myocardial expression of different genes. Increased nitro-oxidative stress, cardiomyocyte hypertrophy, profibrotic signaling, and myocardial remodeling along with apoptotic processes have been reported to play a critical role in the development of cardiomyopathy in both types of DM [15]. Persistent hyperglycemia in diabetes provokes excessive production of reactive oxygen species (ROS) and inflammation which play a key role in diabetic complications $[16,17]$. Hypergly- cemia induces glucose auto-oxidation and surplus generation of ROS. Hyperlipidemia can also increase ROS production through stimulating nicotinamide adenine dinucleotide phosphate (NADPH) oxidases and inducing leakage of the mitochondrial electron transport chain [18]. Excess ROS activates protein kinase $\mathrm{C}$ and subsequently nuclear factor kappa $\mathrm{B}$ (NF$\kappa \mathrm{B})$, leading to myocardial injury $[16,17]$. NF- $\kappa \mathrm{B}$ is a redoxsensitive protein complexes with a central role in inflammation. Activated NF- $\mathrm{BB}$ promotes the transcription and release of inflammatory mediators such as tumor necrosis factor-alpha (TNF- $\alpha$ ) and thereby provokes myocardial inflammation [19].

Metformin is one of the well-known drugs used in type 2 DM and has shown advantages in controlling hyperglycemia and decreasing microvascular complications. However, its role in opposing cardiovascular consequences and cardiovascular mortality needs more investigations [4]. Curiously, a clinical study in 2019 has suggested a significant protective role for metformin/curcumin combination against type I DM-associated cardiac complications [20].

Inositol is a six carbon cyclitol existing under nine stereoisomeric forms depending on the spatial orientation of its six hydroxyl groups. It is the predominant form of inositol present in nature and in our food [21]. Myoinositol is a precursor in the phosphatidylinositol cycle and a source of several second messengers including diacylglycerol, which regulates some members of the protein kinase $\mathrm{C}$ family, inositol-1,4,5triphosphate, which modifies intracellular calcium levels, and phosphatidylinositol-3,4,5-biphosphate, that is involved in the signal transduction. It is a component of cell membranes and is an essential nutrient required by the human cells for growth and survival [22].

The present study was conducted to investigate the cardioprotective effect of metformin and myoinositol either alone or in combination in high-fat diet (HFD), streptozotocin (STZ)induced diabetic rats with the following assessments: fasting blood glucose (FBG), insulin level and lipid profile, along with measurement of PPAR $\alpha$ and GLUT-4 expression levels in the skeletal muscles, cardiac levels of troponin $\mathrm{C}$, nuclear factor kappa, TNF- $\alpha$, and finally detect both the histopathological and immunohistochemical changes of the diabetic rats' heart.

\section{Materials and Methods}

\section{Animals' protocols}

This study was approved by Ain Shams University Research Committee, Faculty of Medicine. This study was performed in accordance with the ethical standards of Ain Shams University Research Committee (ethics committee reference number: 00017585).

Thirty adult male Wistar rats, purchased from an animal farm in Helwan, Cairo, Egypt, and initially weighing 150 - 200 $\mathrm{g}$ (age about 10 weeks) were used in the study. Animals were kept at the animal house of the Research Center at the Bilharzial Research Unit, Faculty of Medicine, Ain Shams University, under standard conditions of boarding: $12 \mathrm{~h}$ light/dark cycles, fed on standard chow, and water ad-libitum, throughout the 
whole period of the study ( 7 weeks). Rats were left to acclimatize for a week before any intervention. The animals were randomly divided into five groups, each containing six rats as the following: group 1: control rats received $0.1 \mathrm{M}$ citrate buffer ( $\mathrm{pH} 4.5$ ); group 2: diabetic control rats received HFD and STZ $(40 \mathrm{mg} / \mathrm{kg}$ ) dissolved in $0.1 \mathrm{M}$ citrate buffer ( $\mathrm{pH} 4.5)$; group 3: myoinositol-treated diabetic rats received myoinositol $(50 \mathrm{mg} /$ $\mathrm{kg}$ ) dissolved in water; group 4: metformin-treated diabetic rats received metformin $(150 \mathrm{mg} / \mathrm{kg})$ dissolved in water; group 5: myoinositol + metformin-treated diabetic rats received metformin $(150 \mathrm{mg} / \mathrm{kg})+$ myoinositol $(50 \mathrm{mg} / \mathrm{kg})$ dissolved in water. The test drugs were administered by gavage once daily for 4 weeks. The age of the animals at the beginning of drug administration was about 14 weeks. Myoinositol (Sigma Aldrich, Germany) was supplied as white powder and was dissolved in water. Metformin (Amoun, Egypt) was supplied as white powder and was dissolved in water. The doses were chosen according to the corresponding human average therapeutic doses used by US Department of Health and Human Services, Food and Drug Administration, Center for Drug Evaluation and Research (CDER) [23].

\section{Sample collection}

Blood samples were collected, from the fasting animals, from the tail vein for biochemical analysis and skeletal muscle tissues (gracilis muscle) were excised rapidly and homogenate was prepared for GLUT-4 estimation and RNA isolation after 7 weeks from the beginning of the experiment (diet administration).

\section{Biochemical analysis}

The FBG was measured by glucose assay kit (Sigma-Aldrich, USA) at 7 weeks. Fasting serum insulin (INS) was measured by enzyme-linked immunosorbent assay (ELISA) kit (MyBioSource, USA) after 7 weeks according to the manufacturers' instructions. Lipid profile measurements (TGs, total cholesterol, LDL and HDL serum levels) were done by triglycerides enzymatic assay kit (XpressBio, USA), cholesterol assay kit (BioChain, USA) and HDL assay kit (BioChain, USA), respectively. LDL was calculated using Friedewald equation after 7 weeks from the beginning of the experiment.

\section{Skeletal muscle tissue homogenization}

The skeletal muscle tissue samples were homogenized by suspending in a $2.0 \mathrm{~mL}$ screw cap tube, containing $200 \mu \mathrm{L}$ of phosphate buffer saline (PBS), a single $5 \mathrm{~mm}$ stainless steel bead (Qiagen) was added to each tube and the samples were homogenized at maximum speed $(30 \mathrm{~Hz})$ for 2 min using the Qiagen Tissue Lyser system. Following homogenization, the samples were spun for $1 \mathrm{~min}$ at maximum speed to reduce foaming, and the homogenate was then applied to the filter column for RNA extraction.

\section{Measurement of GLUT-4 concentration from skeletal muscle using ELISA}

Estimation of GLUT-4 was done using rat GLUT4 ELISA kit (Biospes, China) after 7 weeks from the beginning of the experiment.

\section{Total RNA extraction from skeletal muscle tissues}

Total RNA extraction was performed by miRNeasy mini kit (Qiagen, Germany) (cat no. 217004), then the RNA concentration and purity were measured by a NanoDrop 1000 spectrophotometer [24]. Reverse transcription of the extracted RNA was done using miScript II RT kit (cat no. 218161) (Qiagen, Germany) producing cDNA.

\section{Quantitative real-time polymerase chain reaction (qRT- PCR) for PPAR $\alpha$ gene expression in skeletal muscle tissues}

The quantification of levels was amplified from mRNA using a QuantiTect primer assay primer assays (Rn Ppara 1_SG QuantiTect primer assay, cat no: 249900 , assay $\overline{\mathrm{ID}}$ : QT00176575), and the ACTB primer sequence was used as a housekeeper gene. Quantitative PCR was carried out using QuantiTect SYBR Green PCR kit (Qiagen, Germany) (cat no. 204141) according to manufacturer's instructions. All samples were analyzed using the fiveplex Rotor-Gene PCR analyzer (Qiagen, Germany). Forty cycles were used, each with denaturation at $94{ }^{\circ} \mathrm{C}$ for $15 \mathrm{~s}$, annealing at $55^{\circ} \mathrm{C}$ for $30 \mathrm{~s}$, and extension at $70{ }^{\circ} \mathrm{C}$ for $30 \mathrm{~s}$.

The expression of PPAR $\alpha$ was defined based on the cycle threshold $(\mathrm{Ct})$. The relative expression levels of PPAR $\alpha$ were calculated as 2-Ct after normalization to the expression of ACTB content in each examined tissue sample [25]. Melting curves were performed to check specificity of PCR products.

\section{Histopathological processing of the heart}

The heart was dissected longitudinally, and the whole left ventricle was fixed in $10 \%$ neutral buffered formalin, dehydrated in ascending grades of alcohol, cleared in xylol, embedded in paraffin blocks, and cut into $5 \mu \mathrm{m}$ sections. The sections were dewaxed in xylol, rehydrated in descending grades of alcohol, rinsed with distilled water, and stained with hematoxylin and eosin (H\&E) to evaluate the histological structure of the heart and Masson's trichrome stain to visualize the distribution and content of the collagen fibers.

\section{Immunohistochemical study of the heart}

Immunohistochemistry staining was performed with specific primary antibodies, according to the manufacturer's guidelines. Briefly, sections were subjected to target antigen retrieval with a citrate buffer, blocked with serum for $30 \mathrm{~min}$, and then 
incubated with the anti-NF- $\kappa \mathrm{B}$ p105 antibody produced in rabbit (1:50 - 1:100, SAB4501986; Sigma-Aldrich), anti-troponin T antibody, clone 9C2.1 (1:50 dilution, MABT368; Sigma-Aldrich), and anti-TNF- $\alpha$ antibody (1:50 - 1:200, SAB5700627; Sigma-Aldrich) for $1 \mathrm{~h}$. Sections were washed with PBS, then incubated with biotinylated secondary antibodies for $30 \mathrm{~min}$. Negative controls were prepared by omitting the primary antibody step. The site of antigen-antibody binding was visualized by 3,3'-diaminobenzidine (DAB) staining which appeared as dark-brown. Sections were counterstained with hematoxylin for nuclear staining.

\section{Morphometric analysis of heart histological sections}

The area percentage of collagen fibers in Masson's trichromestained sections as well as the area percentage of the protein expression for immunohistochemical analysis (NF- $\kappa \mathrm{B}$, troponin $T$ and $T N F-\alpha$ ) was quantified using image analyzer Leica Q win V.3 program installed on a computer connected to a Leica DM2500 microscope (Wetzlar, Germany).

The image analyzer was first automatically calibrated to change pixels (measurement units produced by the image analyzer) into micrometer units in a way that one pixel was equal to $0.145 \mu \mathrm{m}$. This was done in five different non-overlapping stained sections using objective lens $\times 40$ (five high-power fields, $\times 40 /$ section) from different rats in each group. The reading of each rat was considered as a single variable. The data were then subjected to statistical analysis.

\section{Statistical analysis}

Analysis of variance (ANOVA) followed by the post-hoc and Kruskal-Wallis tests were conducted for the comparison between four groups through Social Science version 20 (SPSS Software, SPSS Inc., Chicago, IL, USA) before graphing. The results were expressed as mean, standard deviation, median and mean rank, and the level of statistical significance was set at $\mathrm{P}<0.05$.

\section{Results}

Myoinositol and metformin combination improved glycemic control, lipid profile and CK-MB in diabetic rats

Mean levels of serum FBG $(\mathrm{mg} / \mathrm{dL})$, insulin $(\mu \mathrm{IU} / \mathrm{mL}), \mathrm{CK}$ MB (mg/dL), total cholesterol (mg/dL), TGs (mg/dL), LDL $(\mathrm{mg} / \mathrm{dL})$ and HDL $(\mathrm{mg} / \mathrm{dL})$ in the different animal groups were estimated. Diabetic group showed a highly significant increase in serum FBG, insulin, CK-MB, total cholesterol, TGs and LDL levels $(305.8 \pm 59.3,18.6 \pm 2,54 \pm 8.6,118.6 \pm 6.4,111.5$ \pm 7.1 , and $64 \pm 2.3$, respectively) compared to control group $(103.6 \pm 11.4,2.6 \pm 0.2,10 \pm 1.7,41 \pm 2.3,37 \pm 2.7$, and $22 \pm$ 3 , respectively, $\mathrm{P}<0.01$ ), while HDL level was significantly lower in the diabetic group $(24.8 \pm 1.1)$ as compared to the control $(36 \pm 1.4)(\mathrm{P}<0.01)$. Myoinositol $(164 \pm 24.1,9.4 \pm$
$0.7,40 \pm 5.4,62 \pm 3.8,54.8 \pm 2.9$, and $33.8 \pm 1.3)$, metformin $(184 \pm 85.6,6.8 \pm 0.8,24.8 \pm 6.8,50.8 \pm 3.9,47.5 \pm 1.3$, and $31.6 \pm 1.9)$ and myoinositol + metformin-treated groups $(174 \pm$ $59.2,3 \pm 0.2,19.4 \pm 1.7,39.1 \pm 1.5,34.6 \pm 2.1$, and $21.1 \pm 1.9)$ showed significant reduction of FBG, insulin, CK-MB, total cholesterol, TGs and LDL levels compared to diabetic group $(\mathrm{P}<0.01)$; however, myoinositol $(32.3 \pm 0.8)$, metformin $(33.5$ $\pm 0.5)$ and myoinositol + metformin-treated $(36 \pm 0.8)$ groups showed significant increase in HDL level compared to the diabetic group $(\mathrm{P}<0.01)$. Myoinositol + metformin treatment significantly improved the fasting insulin level and lipid profile in diabetic rats better than treatment with either drug alone $(\mathrm{P}<$ 0.01 ) as represented in Table 1.

\section{GLUT-4 protein level increased in skeletal muscle cells after treatment with myoinositol and metformin combina- tion}

In this experiment, ELISA was performed for detecting the concentration of GLUT-4 $(\mu \mathrm{g} / \mathrm{g})$ in skeletal muscle cells in the different animal groups. The mean levels of GLUT-4 in skeletal muscle cells in the diabetic group showed a significant decrease compared to the control $(90.3 \pm 10.1$ and $137.7 \pm 6.8$, respectively, $\mathrm{P}<0.05)$. However, treatment with myoinositol, metformin and both drugs combined together resulted in a significant increase of GLUT-4 levels $(162.1 \pm 32.4,167.5 \pm 37.8$, and $190 \pm 20.5$, respectively) compared to the diabetic animals as shown in Figure 1A.

PPARa overexpressed in skeletal muscle tissue of diabetic rats treated with myoinositol and metformin combination

In this experiment, qRT-PCR was performed for detecting any possible changes in PPAR $\alpha$. The mRNA levels in skeletal muscle tissue homogenate were prepared from the different test groups. The relative quantification (RQ) of PPAR $\alpha$ in skeletal muscle cells in diabetic group (median RQ 0.33) showed a significant decrease compared to the control (median RQ 21.38). On the other hand, myoinositol treatment (median RQ 5.74), metformin treatment (median RQ 17.19) and treatment with both myoinositol and metformin (median RQ 16.22) resulted in a significant increase of PPAR $\alpha$ expression compared to the diabetic group (median RQ 0.33). Combined treatment significantly increased PPAR $\alpha$ expression more than myoinositol treatment only $(\mathrm{P}<0.01$, Fig. 1B).

\section{Negative correlation of GLUT-4 protein and PPAR $\alpha$ mRNA with different biochemical parameters}

There was non-significant direct correlation between GLUT-4 and PPAR $\alpha$ expression levels $(\mathrm{r}=0.34$ with $\mathrm{P}=0.063)$.

GLUT-4 protein $(\mu \mathrm{g} / \mathrm{g})$ and PPAR $\alpha$ mRNA expression (RQ) had a significant inverse correlation with plasma insulin level $(\mu \mathrm{IU} / \mathrm{mL})$, total blood cholesterol $(\mathrm{mg} / \mathrm{dL})$, TGs $(\mathrm{mg} /$ $\mathrm{dL})$, and LDL $(\mathrm{mg} / \mathrm{dL})$, while there was a significant direct 


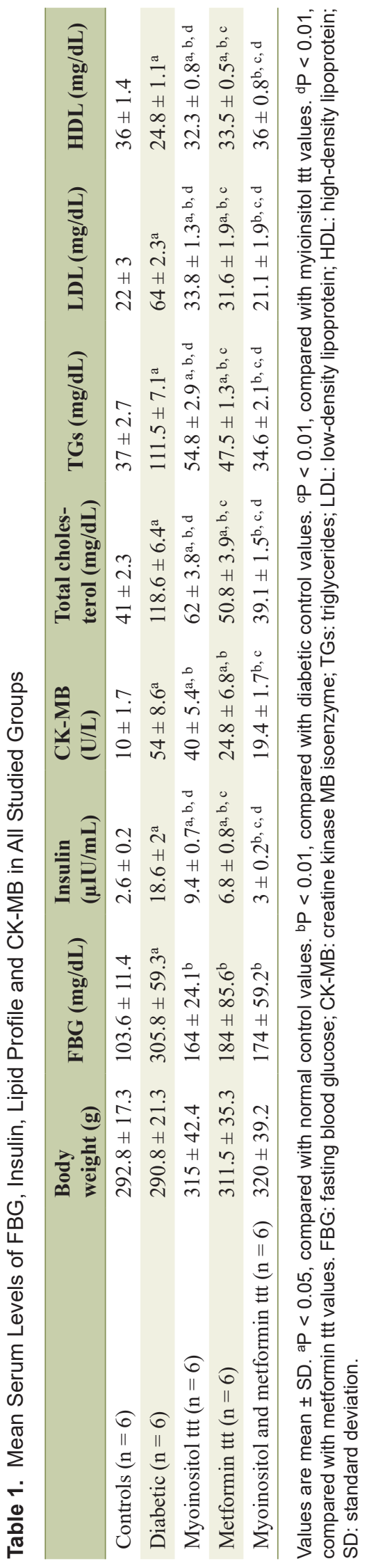

correlation with HDL levels $(\mathrm{mg} / \mathrm{dL})(\mathrm{P}<0.01)$ as shown in Table 2 .

Heart histopathological picture improved after combined
intake of both myoinositol and metformin in diabetic rats

Examination of H\&E-stained sections revealed that the myocardium of the diabetic rats showed deterioration of its architecture as compared to the control group; there were loss of striations, fatty infiltration together with areas of focal degeneration. Furthermore, the cardiac muscle fibers were widely separated from each other by inflammatory cell infiltrations, edema, hemorrhage and dilated congested blood vessels (Fig. $2 \mathrm{~b}, \mathrm{c})$. Myoinositol-treated group showed some improvement in the myocardium architecture as compared to the diabetic group; however, some myocardial cells still appeared deeply acidophilic, and others showed focal necrosis (Fig. 2d). Metformin-treated group also showed some improvement apart from intramyocardial fat infiltration (Fig. 2e). However, treatment of diabetic rats with both metformin and myoinositol resulted in marked improvement of the myocardial structure as compared to the control normal rats with no evidence of degeneration, necrosis, or inflammation (Fig. 2f).

\section{Myoinositol and metformin combination decreased myo- cardial fibrosis in HFD fed STZ-induced diabetic rats}

As presented in Figures 3 and 4A, the Masson's trichromestained sections revealed apparent fibrosis (\%) within the myocardium in the diabetic group. The collagen area percentage was measured as $2.77 \pm 0.83$, which was significantly increased as compared to the control group $(\mathrm{P}<0.05)$ while myoinositol treatment and metformin treatment showed few collagen fibers in between cardiac muscle fibers which resulted in a significant decrease in the collagen area percentage (1.62 \pm 0.89 and $1.05 \pm 0.83$, respectively) as compared to diabetic rats. However, the collagen area percentage was significantly decreased when diabetic rats were treated with both myoinositol and metformin as compared to treatment with either myoinositol or metformin alone $(0.53 \pm 0.16)(\mathrm{P}<0.01)$.

Myoinositol and metformin combination improved cardiac troponin $T$ with down-regulation of NF- $\mathrm{KB}$ p105 and TNF- $\alpha$ in the heart of the diabetic rats

Immunostaining for troponin $\mathrm{T}(\%)$ showed marked positive reaction in normal myocardium as shown in Figures 4B and 5. However, it showed decreased staining in necrotic myocardium in the diabetic rats $(25.31 \pm 2.89)$ as compared to the control group $(61.9 \pm 4.9)(\mathrm{P}<0.05)$. Treatment of both antidiabetic drugs markedly improved the positive immunostaining of cardiac troponin $\mathrm{T}(53.96 \pm 4.29)$ as compared to treatment of each drug alone ( $35 \pm 3.4$ and $32.2 \pm 4.8$, respectively) $(\mathrm{P}<0.01)$.

Immunohistochemical staining for the inflammatory markers (NF- $\mathrm{KB}$ p105 and TNF- $\alpha$ ) in the heart was performed in all 

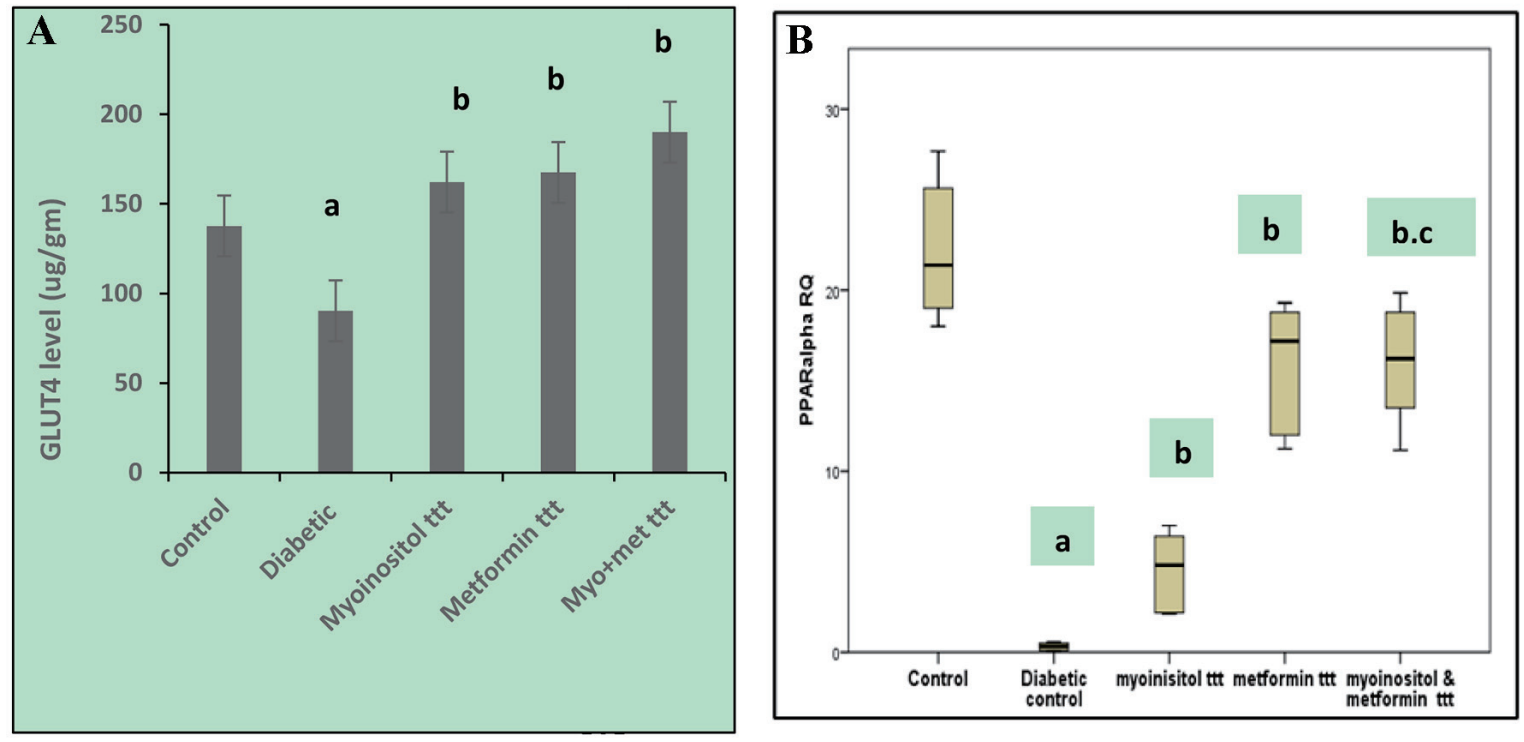

Figure 1. (A) Effect of tested drugs on GLUT-4 concentrations in skeletel muscle cells in HFD-fed STZ-induced diabetic rats. aP $<0.05$, compared with normal control values. ${ }^{\text {bP }}<0.05$, compared with diabetic control values. (B) Box plot showing the RQs of liver tissue PPARa gene mRNA in different groups of the study. The data represent the median fold changes. aP $<0.05$, compared with normal control values. ${ }^{\mathrm{b}} \mathrm{P}<0.05$, compared with diabetic control values. ${ }^{\mathrm{c}} \mathrm{P}<0.05$, compared with myoinositol ttt value. GLUT-4: glucose transporter 4; HFD: high-fat diet; STZ: streptozotocin; RQ: relative quantification; PPARa: peroxisome proliferator-activated receptor-alpha.

groups as shown in Figures 5 and 6. Low expression of NF-kB p105 and TNF- $\alpha(\%)$ was observed in the heart of the control group, and this was quantified as area percentage of protein expression (median 0.5 and 0.3 , respectively). Diabetic rats showed marked positive cytoplasmic reaction for NF- $\mathrm{kB}$ and TNF- $\alpha$ that represented a highly significant increase as compared to the control group (median 7.2 and 1.3, respectively) $(\mathrm{P}<0.05)$. Treatment with both myoinositol and metformin resulted in a high significant decrease in NF- $\mathrm{\kappa B}$ reaction and TNF- $\alpha$ expression (median 0.46 and 0.04 , respectively) than treatment with metformin alone (median 0.64 and 0.15 , respectively) $(\mathrm{P}<0.01$ ).

\section{Discussion}

Development of insulin resistance characterizes the key sys- temic abnormality in the pathogenesis of type 2 DM [26]. Cardiovascular diseases are one of the most common diabetesrelated complications. They generally affect nearly $32.2 \%$ of type $2 \mathrm{DM}$ patients and represent the main cause of death in them [27].

In the current study, we established HFD, STZ-induced diabetic rat model to assess the effect of metformin and/or myoinositol on PPAR $\alpha$ and GLUT-4 expression levels and their correlation with FBG, insulin level and lipid profile.

The results of the present work exhibited that administration of metformin in combination with myoinositol improved insulin resistance as shown by the significant reduction of FBG, insulin, CK-MB, total cholesterol, TGs and LDL levels, and the significant increase in HDL level versus diabetic group as well as the significant rise of GLUT-4 levels and PPAR $\alpha$ expression.

Table 2. Correlations Between GLUT-4 Level, PPARa RQs, FBG, Insulin Levels and Lipid Profile Among All the Studied Groups

\begin{tabular}{|c|c|c|c|c|c|c|}
\hline & FBG (mg/dL) & $\begin{array}{l}\text { Plasma insulin } \\
\text { level }(\mu \mathrm{IU} / \mathrm{mL})\end{array}$ & $\begin{array}{l}\text { Total choles- } \\
\text { terol (mg/dL) }\end{array}$ & $\begin{array}{l}\text { Triglyceride } \\
(\mathrm{mg} / \mathrm{dL})\end{array}$ & $\begin{array}{l}\text { HDL } \\
(\mathrm{mg} / \mathrm{dL})\end{array}$ & LDL (mg/dL) \\
\hline \multicolumn{7}{|l|}{ GLUT-4 $(\mu \mathrm{g} / \mathrm{g})$} \\
\hline Sig. (two-tailed) & 0.260 & 0.011 & 0.001 & 0.001 & 0.003 & 0.005 \\
\hline \multicolumn{7}{|l|}{ PPAR $\alpha$ RQs } \\
\hline
\end{tabular}

Data are presented as r-value. Person's correlation test was used. ${ }^{*}$ Correlation is significant at the 0.05 level (two-tailed). ${ }^{* *}$ Correlation is significant at the 0.01 level (two-tailed). GLUT-4: glucose transporter 4; PPARa: peroxisome proliferator-activated receptor-alpha; RQs: relative quantifications; FBG: fasting blood glucose; HDL: high-density lipoprotein; LDL: low-density lipoprotein. 

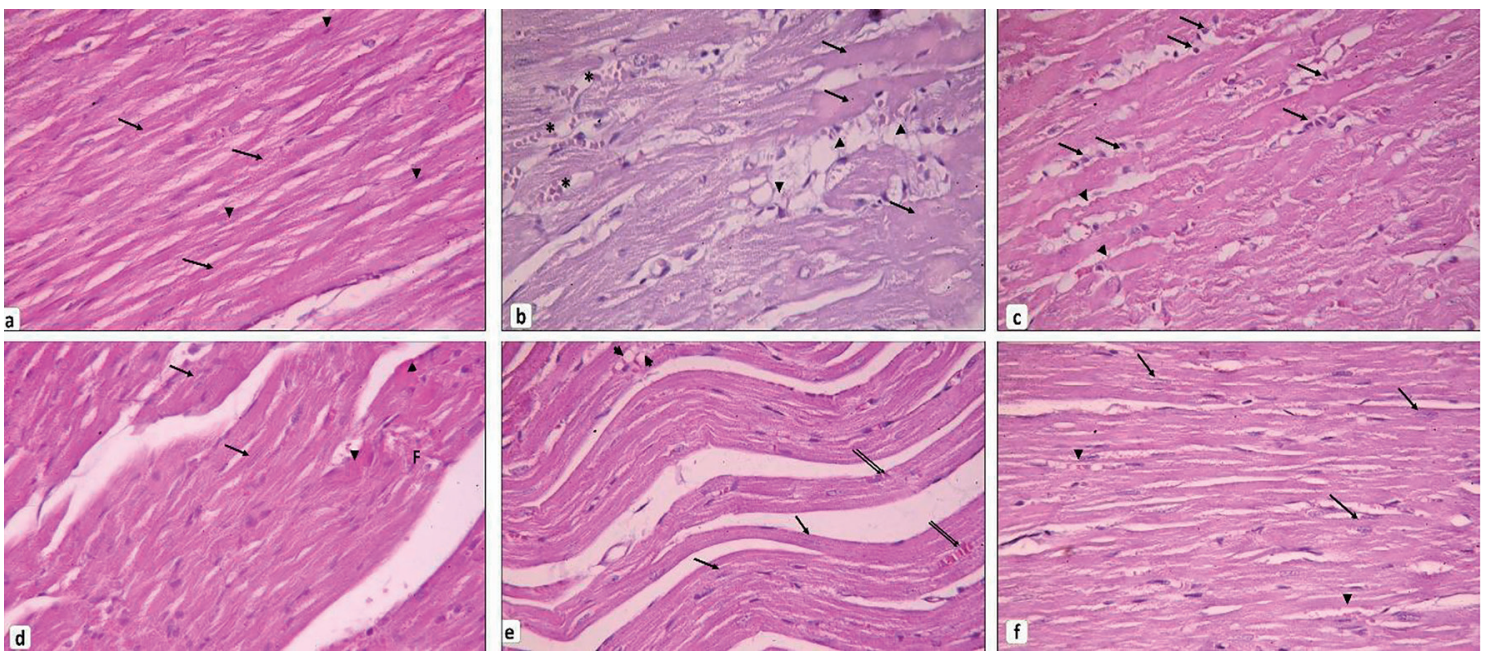

Figure 2. Cardiac muscle histopathology. (a) Control group showing branching and anastomosing cardiac muscle fibers running in different directions. Cardiomyocytes exhibit transverse striations in their sarcoplasm (arrows). Notice the oval vesicular central nuclei of the cardiomyocytes (arrowheads). (b, c) Diabetic group. (b) Loss of striation in some cardiomyocytes and appear with dark acidophilic sarcoplasm devoid of nuclei (arrows). Other cardiac muscle cells are fragmented and infiltrated by fat (arrowheads). Blood vessels in between cardiac muscle appear dilated and congested $\left(^{*}\right)$. (c) Thinned out cardiomyocytes can be seen (arrowheads). Mononuclear cells infiltrate the endomysium in between the widely separated cardiac muscle fibers (arrows). (d) Myoinositol-treated group showing many cardiomyocytes are seen with vesicular nuclei (arrows) and few with deeply acidophilic cytoplasm (arrowheads). Others still exhibit focal fragmentation (F). (e) Metformin-treated group showing many cardiomyocytes are striated with vesicular nuclei (arrows). Others appear discontinuous and infiltrated by fat (arrowheads). Few congested blood vessels can be observed (double arrows). (f) Myoinositol + metformin-treated group showing most cardiomyocytes appear striated with vesicular oval nuclei (arrows). Small, congested blood vessels can be noticed (arrowheads) (H\&E, $\times 400)$. H\&E: hematoxylin and eosin.

For many decades, insulin resistance and type 2 DM have been studied and as well known, insulin resistance is a predictor of potential development of type 2 DM [28].

Insulin resistance and type $2 \mathrm{DM}$ are associated with many dyslipidemia features including reduced HDL cholesterol, increased levels of LDL, TGs, total cholesterol and very lowdensity lipoprotein that are associated with an increased risk of atherosclerotic cardiovascular disease [29, 30].

In the present work, there were significant hypertriglyceridemia, hypercholesterolemia, elevation of LDL cholesterol and reduction of HDL cholesterol in the diabetic group compared to the control group. The antihyperlipidemic of myoinositol and metformin in this study was observed by the significant reduction of both total and LDL cholesterol as well as
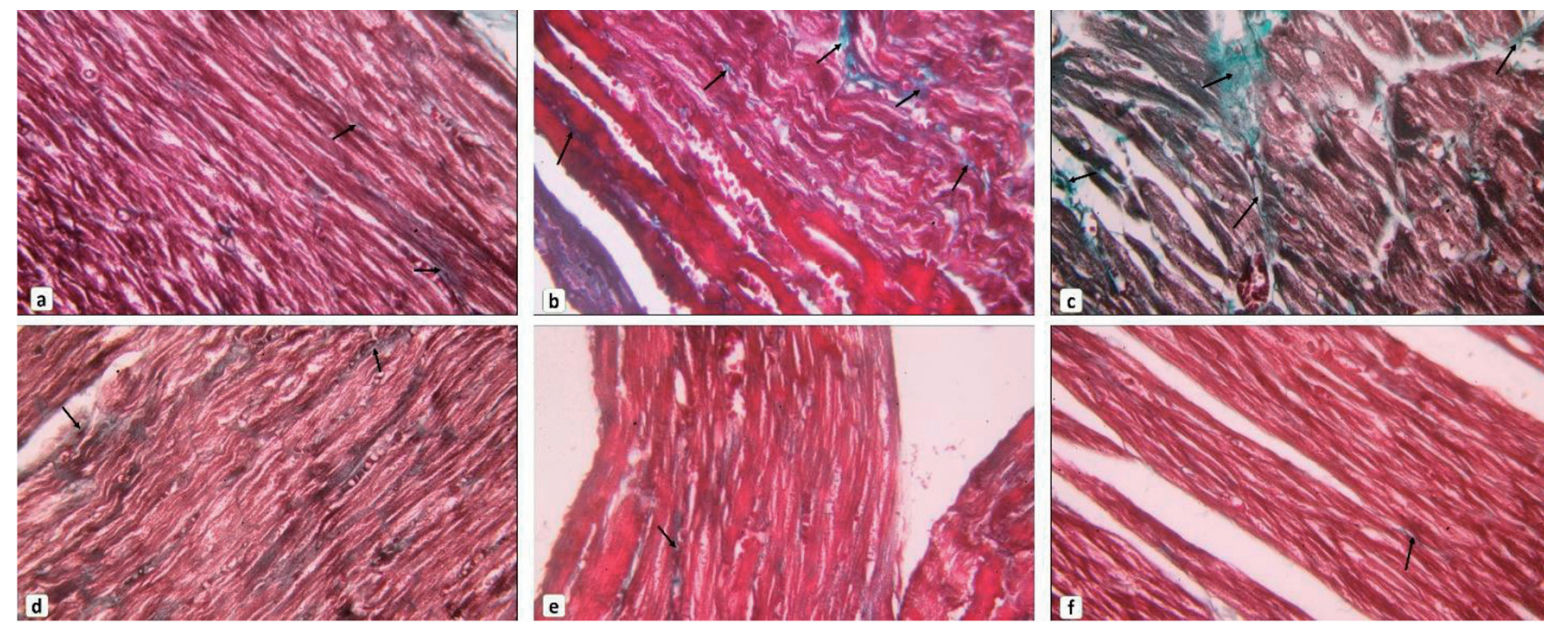

Figure 3. Collagen fibers content in cardiac muscle. (a) Control group, (b, c) diabetic group, (d) myoinositol-treated group, (e) metformin-treated group, (f) myoinositol + metformin-treated group showed most cardiomyocytes appear striated with vesicular oval nuclei (arrows). Small, congested blood vessels can be noticed (arrowhead). Representative images of Masson's trichromestained sections of the myocardium showing collagen fibers distribution in between cardiomyocytes. Arrows indicate content of collagen fibers (Masson's trichrome stain, $\times 400$ ). 

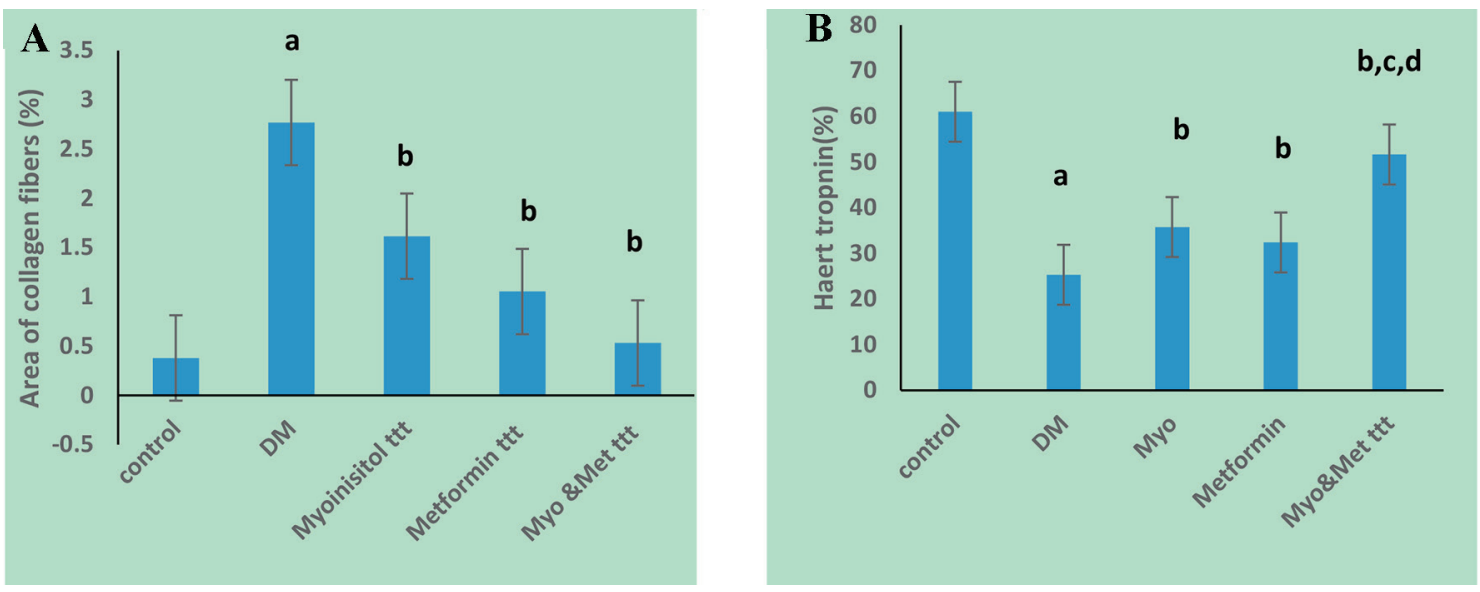

Figure 4. Effect of tested drugs on myocardial fibrosis and troponin T precentage area in cardiac muscle cells in HFD-fed STZinduced diabetic rats. (A) Diabetic group has a significantly the highest collagen percentage area. (B) Diabetic group has a significantly the lowest troponin positive cells while highest percentage in both myoinositol and metformin treated group is observed. ap $<0.01$, compared with normal control values. ${ }^{\mathrm{b}} \mathrm{P}<0.01$, compared with diabetic control values. ${ }^{\mathrm{c} P}<0.01$, compared with myoinositol, dP $<0.01$, compared metformin ttt. HFD: high-fat diet; STZ: streptozotocin.

TGs and elevation of the HDL level. Our results showed that combined treatment with both drugs had a more lipid lowering effect than each alone, and this effect was additive and better regarding the lipid profile control.

These results were in accordance with the studies by Muscogiuri et al and Iuorno et al, who found that systemic myoinositol supplementation improved systolic blood pressure, insulin resistance, cholesterol, and TGs levels in postmenopausal women with metabolic syndrome [31, 32]. Our results were also concomitant with the study by Karamchand et al, whose results clearly indicated the beneficial effect of metformin on lipid profile of type $2 \mathrm{DM}$, hence concluding that metformin had the ability to correct dyslipidemia in type 2 diabetic patients [30].

Expression of GLUT-4 was noticed in the tissues possessing insulin stimulated glucose transport as adipose tissues and skeletal and cardiac muscle as detected by Szablewski et al [33]. Knockout study of skeletal muscle and adipose tissue specific GLUT-4 mice showed severe insulin resistance and glucose intolerance at an early age [34].

Our results showed that the level of GLUT-4 was significantly lowered in the diabetic rats compared to the control, yet

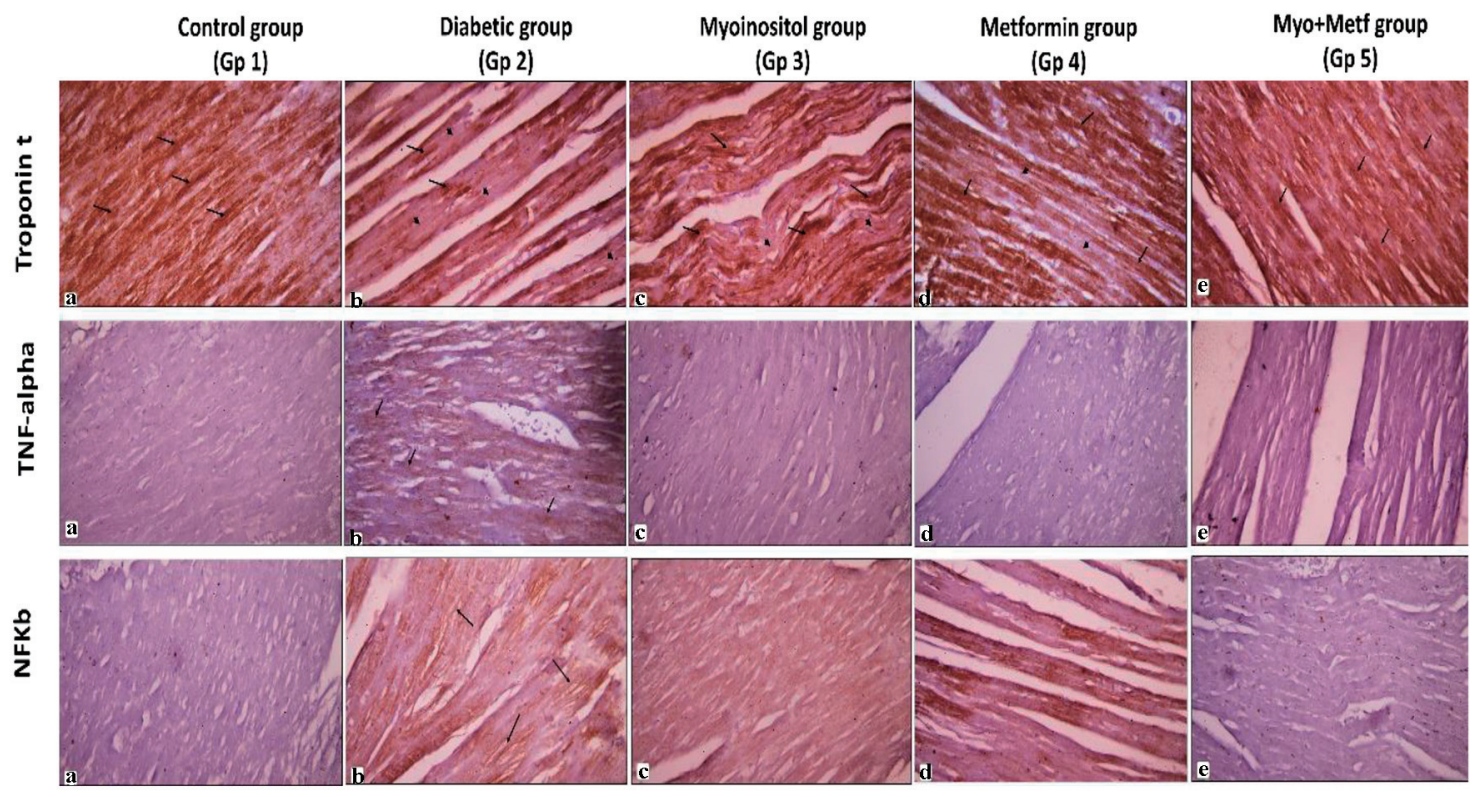

Figure 5. Immunohistochemical stains of the cardiac muscle. Representative immunoperoxidase images showing expression of troponin T, TNF- $\alpha$ and NF-KB p105 in the myocardium of normal, diabetic and the three treated groups. Arrows indicate positive brown sites where the immune marker is expressed. Arrowheads indicate areas of negative immune reaction (avidin-biotin peroxidase, $\times 400)$. TNF- $\alpha$ : tumor necrosis factor alpha; NF-kB: nuclear factor kappa B. 

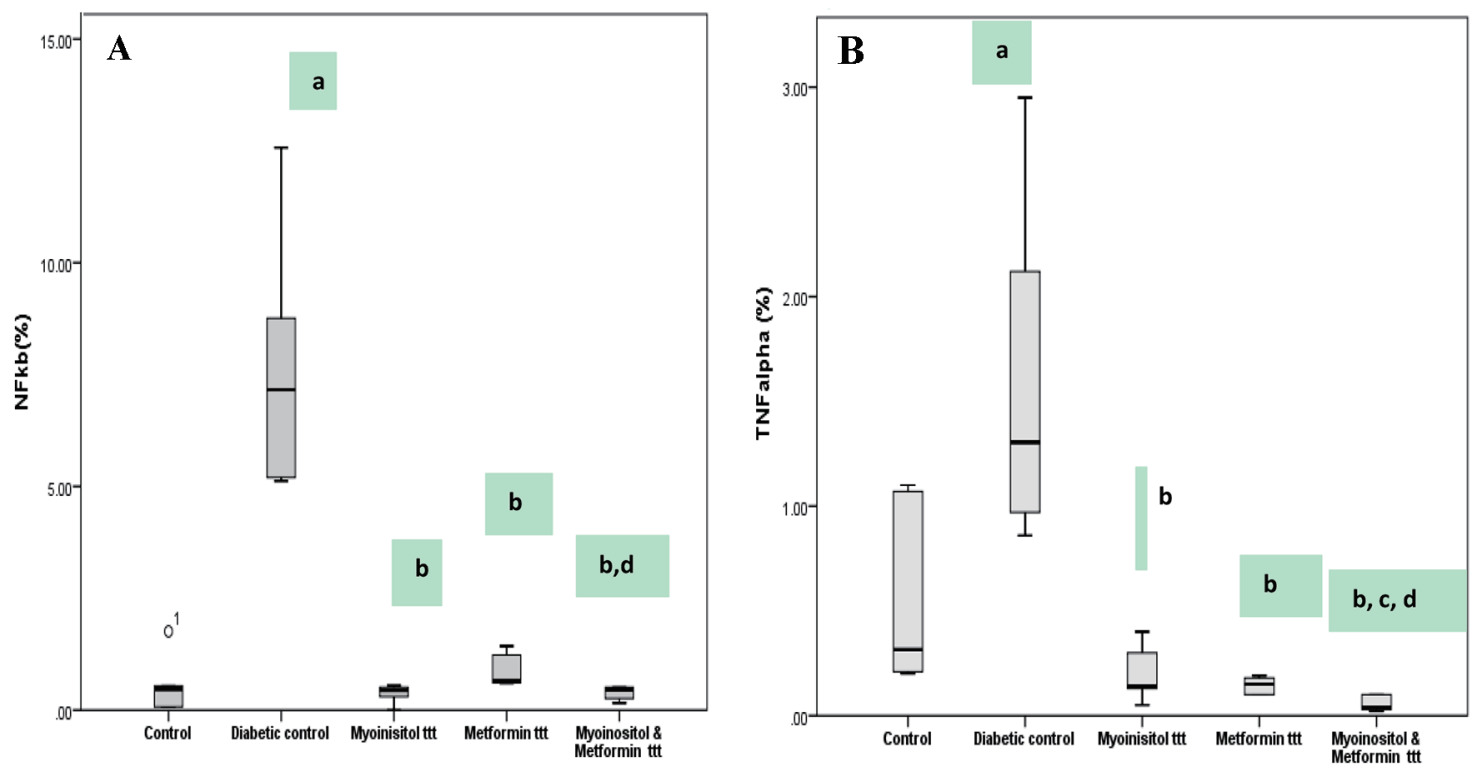

Figure 6. Box plot represents expression of NF-KB and TNF- $\alpha$ detected by immunohistochemical staining among different groups. (A) Diabetic group has the highest NF-KB percentage area and stain intensity while lowest percentage in both myoinositol and metformin-treated group is observed. (B) Diabetic group has the highest TNF- $\alpha$ percentage positive cells while lowest percentage in both myoinositol and metformin-treated group is observed. aP $<0.01$, compared with normal control values. ${ }^{\text {bP }}<$ 0.01 , compared with diabetic control values. ${ }^{\mathrm{c} P}<0.01$, compared with myoinositol. ${ }^{\mathrm{d} P}<0.01$, compared metformin ttt. TNF- $\alpha$ : tumor necrosis factor alpha; NF-kB: nuclear factor kappa B.

its level increased with treatment with either myoinositol or metformin and was more pronounced with combined myoinositol and metformin treatment, resulting from the improvement of insulin sensitivity by the combined treatment. Metformin could reduce hyperglycemia also by improving peripheral insulin sensitivity and increasing glucose uptake into skeletal muscle cells [35]. On the other hand, Lee et al reported that metformin chronically induced Cbl-associated protein expression via AMP-activated protein kinase, and modulated GLUT4 translocation [36].

The current results regarding myoinositol agreed with the study by Bevilacqua and Bizzarri, who found that both types of inositol phosphoglycans (containing myoinositol and D-chrio inositol) acted as second messengers downstream of insulin receptors, exerting an insulin mimetic activity. When administered to normal or diabetic rats, they reduced hyperglycemia in a dose-dependent fashion and encouraged muscular glycogenesis [37].

Since both metformin and myoinositol could increase GLUT-4 by different mechanisms, their effect could be additive as observed in our work.

The current study found that PPAR $\alpha$ was down expressed in the diabetic rats as compared to both the control and treated groups. Interestingly, PPAR $\alpha$ expression was significantly upregulated after treatment with metformin and myoinositol combined together as compared to either metformin or myoinositol alone. Moreover, the overexpression of PPAR $\alpha$ was associated with decrease in TGs, total cholesterol and LDL levels and increase in HDL levels. Therefore, this study postulated that combined treatment with both metformin and myoinositol increased insulin sensitivity and improved lipid profile through upregulation of GLUT-4 and PPAR $\alpha$ receptors expression in skeletal muscles. Many evidences had indicated that hypertriglyceridemia caused an excess of free fatty acids, which could increase insulin resistance "lipotoxicity" that is a vital pathogenetic issue clearly related with peripheral tissue insulin resistance and islet $\beta$-cell dysfunction [38]. Feng et al reported that PPAR $\alpha$ agonists may protect against the cardiovascular complications of prediabetes and DM through decreasing hypertriglyceridemia, improving insulin sensitivity and $\beta$-cell function [39].

Furthermore, this study elucidates the protective role of this drug combination against the development of cardiac injury in diabetic rats. We evaluated the cardiac levels of troponin $\mathrm{C}$, nuclear factor kappa, TNF- $\alpha$, and the histopathological changes that occurred in the cardiac muscles. We revealed that the combined treatment with both myoinositol and metformin triggered marked improvement of the cardiac histopathological changes as detected by the significant improvement of the myocardial structure and the significant decrease of degeneration, necrosis, or inflammation and the collagen area percentage against diabetic group. A marked improvement of the positive immunostaining of cardiac troponin $T$ with significant decrease in NF- $\kappa \mathrm{B}$ and TNF- $\alpha$ expression was observed.

Histopathological changes of the myocardium of the diabetic rats showed areas of necrosis, fatty infiltrations, mononuclear cellular infiltrations, and congestion. The improvement of the cardiac architecture in the present study was better in combined metformin and myoinositol treatment than in metformin or myoinositol-treated groups. These results were in accordance with Roslan et al, who suggested the occurrence of cardiomyopathy induced by diabetes [40]. Hyperglycemia or 
unstable glycemic metabolism leads to activation of leukocyte and recruitment of inflammatory neutrophils, monocytes, and macrophages to the myocardium [41].

Additionally, the accumulation of collagen fibers in the heart interstitium is another common finding of diabetic cardiomyopathy resulting in the inability of the heart to contract and relax efficiently [42]. In the current work, myocardial fibrosis was noticed in the diabetic group, consistent with other studies in different animal models of diabetes [43]. The deposition of collagen fibers was significantly decreased in combined treatment of metformin and myoinositol suggesting their coordinated role in preventing diabetes-induced interstitial fibrosis. Levick and Widiapradja postulated that hyperglycemia induced activation of many signaling pathways including renin angiotensin system, kinases, cytokines, and non-coding RNA. These signaling pathways resulted in activation of TGF- $\beta 1$ causing excessive secretion of extracellular matrix especially collagen type I by cardiac fibroblasts [44].

Our results displayed areas with loss of immune expression of cardiac troponin $\mathrm{T}$ ( $\mathrm{cTnT}$ ) in the heart of the diabetic rats indicating focal myocardial necrosis. Treatment with combined metformin and myoinositol demonstrated marked significant increase in immune reaction for cTnT. Fishbein et al designated that immunohistochemical staining for cTnI and cTnT might be more sensitive than routine H\&E staining for the diagnosis of myocardial necrosis in experimental animals as well as autopsy of human hearts [45].

In addition, many studies indicated that cardiomyopathy associated with DM results from myocardial inflammation. These proinflammatory responses and chronic low-grade inflammation that happen within cardiomyocytes are reactions to hyperglycemia and dyslipidemia that take place in insulin resistance and DM [46] as hyperglycemia triggers the production of pro-inflammatory cytokines such as interleukin$1 \beta$ (IL-1 $\beta$ ), interleukin-6 (IL-6), and TNF- $\alpha$, through activating the $\mathrm{NF}-\kappa \mathrm{B}$ signaling pathway [47]. In the present study, it was observed that immunostaining for TNF- $\alpha$ was significantly increased in the myocardium of the diabetic rats and thereby activating the NF- $\mathrm{BB}$ signaling cascade which showed also increased immune reactions as compared to the control group. Administration of both antidiabetic drugs in combination could inhibit this increase in TNF- $\alpha$ and NF$\kappa \mathrm{B}$ immune reactivity in the cardiac tissue, thus ameliorating cardiac inflammation. Similarly, Saklani et al proposed that TNF- $\alpha$ is essential for pathogenesis of diabetic cardiomyopathy [48].

\section{Conclusion}

Adding myoinositol to metformin was associated with GLUT4 and PPAR $\alpha$ overexpression in skeletal muscle tissue. This may contribute to increasing insulin sensitivity with better glycemic control and improving dyslipidemia. The combined therapy myoinositol plus metformin could be a promising new strategy to improve the metabolic profile in obese diabetic patients, thus reducing the risk for the various cardiovascular events.

\section{Acknowledgments}

None to declare.

\section{Financial Disclosure}

This research did not receive any specific grant from funding agencies in the public, commercial, or not-for-profit sectors.

\section{Conflict of Interest}

The authors declare that there is no conflict of interest.

\section{Informed Consent}

Not applicable.

\section{Author Contributions}

All authors agreed to be accountable for all aspects of the work in ensuring that questions related to the accuracy or integrity of any part of the work are appropriately investigated and resolved. They have made substantial contributions and roles in this manuscript. Dalia Abdel-Wahab Mohamed, Dina Sayed Abdulrahim, Yomna M. Tamim and Asmaa A. Abozeid have a contribution in conception and design, acquisition of data, analysis and interpretation of data and drafting the manuscript. Wael M. Elayat, Mohamed F. Abdel-Salam, and Nahla Mohamed Teama contributed to analysis, interpretation of data and revising.

\section{Data Availability}

The data supporting the findings of this study are available from the corresponding author upon reasonable request.

\section{References}

1. Diabetes Canada Clinical Practice Guidelines Expert Committee, Punthakee Z, Goldenberg R, Katz P. Definition, classification and diagnosis of diabetes, prediabetes and metabolic syndrome. Can J Diabetes. 2018;42(Suppl 1):S10-S15.

2. Chamberlain JJ, Herman WH, Leal S, Rhinehart AS, Shubrook JH, Skolnik N, Kalyani RR. Pharmacologic therapy for type 2 diabetes: synopsis of the 2017 American Diabetes Association standards of medical care in diabetes. Ann Intern Med. 2017;166(8):572-578.

3. Haththotuwa R. Cardiovascular complications of diabetes. InnovAiT [Internet]. 2016;9(11):694-701.

4. Zhang K, Yang W, Dai H, Deng Z. Cardiovascular risk 
following metformin treatment in patients with type 2 diabetes mellitus: Results from meta-analysis. Diabetes Res Clin Pract. 2020;160:108001.

5. Davies MJ, D'Alessio DA, Fradkin J, Kernan WN, Mathieu C, Mingrone G, Rossing P, et al. Management of Hyperglycemia in Type 2 Diabetes, 2018. A consensus report by the American Diabetes Association (ADA) and the European Association for the Study of Diabetes (EASD). Diabetes Care. 2018;41(12):2669-2701.

6. Huang S, Czech MP. The GLUT4 glucose transporter. Cell Metab. 2007;5(4):237-252.

7. Gutierrez-Rodelo C, Roura-Guiberna A, Olivares-Reyes JA. [Molecular mechanisms of insulin resistance: an update]. Gac Med Mex. 2017;153(2):214-228.

8. Boitier E, Gautier JC, Roberts R. Advances in understanding the regulation of apoptosis and mitosis by peroxisome-proliferator activated receptors in pre-clinical models: relevance for human health and disease. Comp Hepatol. 2003;2(1):3.

9. Willson TM, Brown PJ, Sternbach DD, Henke BR. The PPARs: from orphan receptors to drug discovery. J Med Chem. 2000;43(4):527-550.

10. Neschen S, Morino K, Dong J, Wang-Fischer Y, Cline GW, Romanelli AJ, Rossbacher JC, et al. n-3 Fatty acids preserve insulin sensitivity in vivo in a peroxisome proliferator-activated receptor-alpha-dependent manner. Diabetes. 2007;56(4):1034-1041.

11. Fruchart JC, Staels B, Duriez P. The role of fibric acids in atherosclerosis. Curr Atheroscler Rep. 2001;3(1):83-92.

12. Ribet C, Montastier E, Valle C, Bezaire V, Mazzucotelli A, Mairal A, Viguerie N, et al. Peroxisome proliferatoractivated receptor-alpha control of lipid and glucose metabolism in human white adipocytes. Endocrinology. 2010;151(1):123-133.

13. Botta M, Audano M, Sahebkar A, Sirtori CR, Mitro N, Ruscica M. PPAR Agonists and Metabolic Syndrome: An Established Role? Int J Mol Sci. 2018;19(4):1197.

14. Hong F, Xu P, Zhai Y. The opportunities and challenges of peroxisome proliferator-activated receptors ligands in clinical drug discovery and development. Int J Mol Sci. 2018;19(8):2189.

15. Huynh K, Bernardo BC, McMullen JR, Ritchie RH. Diabetic cardiomyopathy: mechanisms and new treatment strategies targeting antioxidant signaling pathways. Pharmacol Ther. 2014;142(3):375-415.

16. Khanra R, Dewanjee S, T KD, Sahu R, Gangopadhyay M, De Feo V, Zia-Ul-Haq M. Abroma augusta L. (Malvaceae) leaf extract attenuates diabetes induced nephropathy and cardiomyopathy via inhibition of oxidative stress and inflammatory response. J Transl Med. 2015;13:6.

17. Bhattacharjee N, Barma S, Konwar N, Dewanjee S, Manna P. Mechanistic insight of diabetic nephropathy and its pharmacotherapeutic targets: An update. Eur J Pharmacol. 2016;791:8-24.

18. Steinberg HO, Paradisi G, Hook G, Crowder K, Cronin J, Baron AD. Free fatty acid elevation impairs insulin-mediated vasodilation and nitric oxide production. Diabetes. 2000;49(7):1231-1238.

19. Shah MS, Brownlee M. Molecular and Cellular Mecha- nisms of Cardiovascular Disorders in Diabetes. Circ Res. 2016;118(11):1808-1829.

20. Abdelsamia EM, Khaleel SA, Balah A, Abdel Baky NA. Curcumin augments the cardioprotective effect of metformin in an experimental model of type I diabetes mellitus; Impact of Nrf2/HO-1 and JAK/STAT pathways. Biomed Pharmacother. 2019;109:2136-2144.

21. Croze ML, Geloen A, Soulage CO. Abnormalities in myo-inositol metabolism associated with type 2 diabetes in mice fed a high-fat diet: benefits of a dietary myoinositol supplementation. Br J Nutr. 2015;113(12):18621875.

22. Carlomagno G, Unfer V. Inositol safety: clinical evidences. Eur Rev Med Pharmacol Sci. 2011;15(8):931-936.

23. Food and Drug Administration. Guidance for industry: estimating the maximum safe starting dose in initial clinical trials for therapeutics in adult healthy volunteers. Cent Drug Eval Res. 2005;7.

24. Mar-Aguilar F, Luna-Aguirre CM, Moreno-Rocha JC, Araiza-Chavez J, Trevino V, Rodriguez-Padilla C, Resendez-Perez D. Differential expression of miR-21, miR$125 \mathrm{~b}$ and miR-191 in breast cancer tissue. Asia Pac J Clin Oncol. 2013;9(1):53-59.

25. Schmittgen TD, Livak KJ. Analyzing real-time PCR data by the comparative $\mathrm{C}(\mathrm{T})$ method. Nat Protoc. 2008;3(6):1101-1108.

26. Roden M, Petersen K, Shulman G. Insulin Resistance in Type 2 Diabetes. In: Textbook of Diabetes [Internet]. John Wiley \& Sons, Ltd; 2017. p. 174-186. Available from: https://onlinelibrary.wiley.com/doi/ abs/10.1002/9781118924853.ch13.

27. Einarson TR, Acs A, Ludwig C, Panton UH. Prevalence of cardiovascular disease in type 2 diabetes: a systematic literature review of scientific evidence from across the world in 2007-2017. Cardiovasc Diabetol. 2018;17(1):83.

28. Taylor R. Insulin resistance and type 2 diabetes. Diabetes. 2012;61(4):778-779.

29. Patel TP, Rawal K, Bagchi AK, Akolkar G, Bernardes N, Dias DDS, Gupta S, et al. Insulin resistance: an additional risk factor in the pathogenesis of cardiovascular disease in type 2 diabetes. Heart Fail Rev. 2016;21(1):11-23.

30. Karamchand S, Leisegang R, Schomaker M, Maartens G, Walters L, Hislop M, Dave JA, et al. Risk factors for incident diabetes in a cohort taking first-line nonnucleoside reverse transcriptase inhibitor-based antiretroviral therapy. Medicine (Baltimore). 2016;95(9):e2844.

31. Muscogiuri G, Palomba S, Lagana AS, Orio F. Inositols in the treatment of insulin-mediated diseases. Int J Endocrinol. 2016;2016:3058393.

32. Iuorno MJ, Jakubowicz DJ, Baillargeon JP, Dillon P, Gunn RD, Allan G, Nestler JE. Effects of d-chiro-inositol in lean women with the polycystic ovary syndrome. Endocr Pract. 2002;8(6):417-423.

33. Szablewski L. Glucose transporters in healthy heart and in cardiac disease. Int J Cardiol. 2017;230:70-75.

34. Abel ED, Peroni O, Kim JK, Kim YB, Boss O, Hadro E, Minnemann T, et al. Adipose-selective targeting of the GLUT4 gene impairs insulin action in muscle and liver. Nature. 2001;409(6821):729-733. 
35. Madiraju AK, Erion DM, Rahimi Y, Zhang XM, Braddock DT, Albright RA, Prigaro BJ, et al. Metformin suppresses gluconeogenesis by inhibiting mitochondrial glycerophosphate dehydrogenase. Nature. 2014;510(7506):542546.

36. Lee JO, Lee SK, Kim JH, Kim N, You GY, Moon JW, Kim SJ, et al. Metformin regulates glucose transporter 4 (GLUT4) translocation through AMP-activated protein kinase (AMPK)-mediated Cbl/CAP signaling in 3T3-L1 preadipocyte cells. J Biol Chem. 2012;287(53):4412144129.

37. Bevilacqua A, Bizzarri M. Inositols in insulin signaling and glucose metabolism. Int J Endocrinol. 2018;2018:1968450.

38. Montecucco F, Steffens S, Mach F. Insulin resistance: a proinflammatory state mediated by lipid-induced signaling dysfunction and involved in atherosclerotic plaque instability. Mediators Inflamm. 2008;2008:767623.

39. Feng X, Gao X, Jia Y, Xu Y. PPAR-alpha agonist fenofibrate reduces insulin resistance in impaired glucose tolerance patients with hypertriglyceridemia: a cross-sectional study. Diabetes Ther. 2017;8(2):433-444.

40. Roslan J, Giribabu N, Karim K, Salleh N. Quercetin ameliorates oxidative stress, inflammation and apoptosis in the heart of streptozotocin-nicotinamide-induced adult male diabetic rats. Biomed Pharmacother. 2017;86:570582.

41. Rao X, Zhong J, Sun Q. The heterogenic properties of monocytes/macrophages and neutrophils in inflammatory response in diabetes. Life Sci. 2014;116(2):59-66.

42. Li G, Yang L, Feng L, Yang J, Li Y, An J, Li D, et al.
Syringaresinol protects against type 1 diabetic cardiomyopathy by alleviating inflammation responses, cardiac fibrosis, and oxidative stress. Mol Nutr Food Res. 2020;64(18):e2000231.

43. Val-Blasco A, Prieto P, Gonzalez-Ramos S, Benito G, Vallejo-Cremades MT, Pacheco I, Gonzalez-Peramato $\mathrm{P}$, et al. NOD1 activation in cardiac fibroblasts induces myocardial fibrosis in a murine model of type 2 diabetes. Biochem J. 2017;474(3):399-410.

44. Levick SP, Widiapradja A. The diabetic cardiac fibroblast: mechanisms underlying phenotype and function. Int J Mol Sci. 2020;21(3):970.

45. Fishbein MC, Wang T, Matijasevic M, Hong L, Apple FS. Myocardial tissue troponins T and I. An immunohistochemical study in experimental models of myocardial ischemia. Cardiovasc Pathol. 2003;12(2):65-71.

46. Filardi T, Ghinassi B, Di Baldassarre A, Tanzilli G, Morano S, Lenzi A, Basili S, et al. Cardiomyopathy associated with diabetes: the central role of the cardiomyocyte. Int J Mol Sci. 2019;20(13):3299.

47. Frati G, Schirone L, Chimenti I, Yee D, Biondi-Zoccai G, Volpe M, Sciarretta S. An overview of the inflammatory signalling mechanisms in the myocardium underlying the development of diabetic cardiomyopathy. Cardiovasc Res. 2017;113(4):378-388.

48. Saklani R, Gupta SK, Mohanty IR, Kumar B, Srivastava $\mathrm{S}$, Mathur R. Cardioprotective effects of rutin via alteration in TNF-alpha, CRP, and BNP levels coupled with antioxidant effect in STZ-induced diabetic rats. Mol Cell Biochem. 2016;420(1-2):65-72. 